\title{
PERFORMANCE OF THE SRRC STORAGE RING AND WIGGLER COMMISSIONING
}

\author{
C.C. Kuo, K.T. Hsu, G.H. Luo, W.K. Lau, Ch. Wang, H.P. Chang, \\ L.H. Chang, M.H. Wang, J.C. Lee, C.S. Hsue, W.T. Weng*, Y.C. Liu ${ }^{\dagger}$ \\ Synchrotron Radiation Research Center \\ No 1. R\&D Rd VI, Hsinchu Science-Based Industrial Park, Hsinchu, Taiwan, R.O.C.
}

\section{Abstract}

A $1.3 \mathrm{GeV}$ synchrotron radiation storage ring at SRRC has been operated for more than a year since October 1993. Starting from April 1994, the machine has been open to the user community. In February 1995, We installed a wiggler magnet of 1.8 tesla 25 -pole in the ring and successfully commissioned. The machine was scheduled for the users' runs from the middle of April this year. We describe the performance of the machine without wiggler magnet system and then report the wiggler effects on the beam dynamics of the storage ring, e.g., tune shift, beta-beating, orbit change, nonlinear dynamics effect, etc. Some measurements are compared with the model prediction and agreement between them was fairly good. Possible actions to minimize wiggler effects have been taken, such as orbit correction as a function wiggler gap change. The machine improvement projects, such as longitudinal and transverse damping systems as well as orbit stability feedback system are under construction and will be in use soon.

\section{INTRODUCTION}

The $1.3 \mathrm{GeV}$ synchrotron radiation light source located in Hsinchu, Taiwan, is a storage ring of emittance $1.9 \times 10^{-8} \mathrm{~m}$ rad and circumference $120 \mathrm{~m}$, which has been operated for the VUV users for more than a year.[1,2] To increase the photon flux in the hard x-ray region of the synchrotron radiation spectrum, a wiggler magnet system was installed and commissioned in the beginning of this year. With such an insertion device, x-ray users would obtain more photon flux at the higher photon energy region.

At users' request, the machine has been scheduled five days per week, 24 hours per day from November 1994. The user beam time is increased to 10 shifts per week. Before installation of the wiggler, the machine was refilled every 8-hour interval to $200 \mathrm{~mA}$. Typical beam lifetime at $200 \mathrm{~mA}$ stored current was 6 hours. We report the machine performance and then result from wiggler commissioning.

\section{MACHINE PERFORMANCE}

We usually run machine in the multibunch operation mode for user beam time with initial stored beam current $200 \mathrm{~mA}$, which is the design value. Typical filling rate was about 5-10 $\mathrm{mA} / \mathrm{s}$. A long empty gap in the bunch train was introduced to

\footnotetext{
* Permanent address: AGS Department, BNL Upton, NY, USA.

$\dagger$ Permanent address: Department of Physics, National Tsing-Hua University, Hsinchu, Taiwan, R.O.C.
}

reduce the ion-related effects. Before we installed a new wiggler vacuum chamber, the base pressure was around $0.4 \mathrm{nTorr}$ and dynamic pressure was about 1-2 nTorr at $200 \mathrm{~mA}$. The Coulomb lifetime was over 20 hours and the lifetime of the stable beam was about 6 hours at $200 \mathrm{~mA}$, which was dominated by the Touschek scattering.

The typical measured horizontal beam size at bending port was about $200-250 \mu \mathrm{m}$ at $200 \mathrm{~mA}$. The increase from the design value $150 \mu \mathrm{m}$ was due to the longitudinal coupled bunch instabilities.[3] Some actions to get rid of such instabilities have been planned, e.g., temperature control of the rf cavities to shift the higher order mode frequencies or implementing longitudinal damping system. In the vertical plan, we observed an emittance coupling about $5 \%$, which was mainly due to spurious vertical dispersion.

Up to now, the single bunch operation mode was only for machine studies. A bunch current up to $30 \mathrm{~mA}$ could be accumulated in a few minutes and bunch purity could be better than $10^{-3}$ with bunch purification process.[4]

We observed severe transverse beam instability, in particular in the vertical plane. At least up to present, this instability was attributed to the beam-ion interaction. To cure this instability, several methods were tried, e.g., increase of ring chromaticity, beam shaking, ion shaking, filling pattern control, cleaning electrodes, and transverse damping system. In practice, only the way by increasing the chromaticity or by implementing a transverse damping system demonstrated its effectiveness and feasibility for the routine operation. We usually run the machine with higher positive chromaticity. In the near future, we will use transverse damping system to cope with such instabilities.[5]

We were able to correct the beam closed orbit down to $0.2 \mathrm{~mm}$ level in both planes.[6] The on-going study to get precise BPM and quadrupole center position together with the improvement of the BPM precision will enable us to obtain more accurate and reduced closed orbit.

The orbit oscillations around 10-15 $\mu \mathrm{m}$ in both planes were detected both with electron BPM and photon BPM. A slow variation of orbit in about $90 \mathrm{sec}$ was correlated with the water temperature change. An orbit feedback system to reduce orbit fluctuation seen by the synchrotron radiation users is under test .[7]

\section{WIGGLER COMMISSIONING}

The structure of the wiggler is a hybrid planar type of which permanent magnetic material is $\mathrm{NdFeB}$ and pole material is Vanadium permendur. The pole period is $20 \mathrm{~cm}$ long and the 
effective pole number is 25 . The peak field strength is more than 1.8 Tesla at the minimum wiggler gap $22 \mathrm{~mm}$. The overall magnet length is about $3.04 \mathrm{~m}$ and located $80 \mathrm{~m}$ downstream of the injection point. The wiggler system was manufactured by STI Optronics in Seattle USA. The magnetic field properties were corrected and carefully measured to meet the specifications.

It took about one month in installing the wiggler system, changing and baking the vacuum chamber as well as adding some diagnostics and correction elements. In the end of February, we succeeded in closing the wiggler gap without any beam loss in the first try of the beam test. The poor vacuum and smaller physical aperture of the newly installed wiggler chamber result in less efficient injection rate and poor beam lifetime. Further optimization of the injection process to reduce the beam loss in the wiggler chamber is under way so as to prevent possible damage of the magnet material with mass radiation bombardment. As a result, during the injection the wiggler gap is still open. The vacuum cleaning with synchrotron radiation is necessary to improve the beam lifetime.

The planar wiggler with $k_{x}=0$ acts like an extra vertical focusing element and no effect in the horizontal plane. Therefore the vertical optics is distorted and vertical tune shall be shifted, whereas the horizontal optics shall be the same as that without wiggler field. We measured the tunes as a function gap and compared with the model prediction. No change in the horizontal tune is observed while the vertical tune shift is in fairly agreement with the model calculation as shown in Fig. 1. The working point was set at $(7.236,4.088)$ when wiggler was open. The change of the working point is still away from the systematic resonance lines up to fifth order.

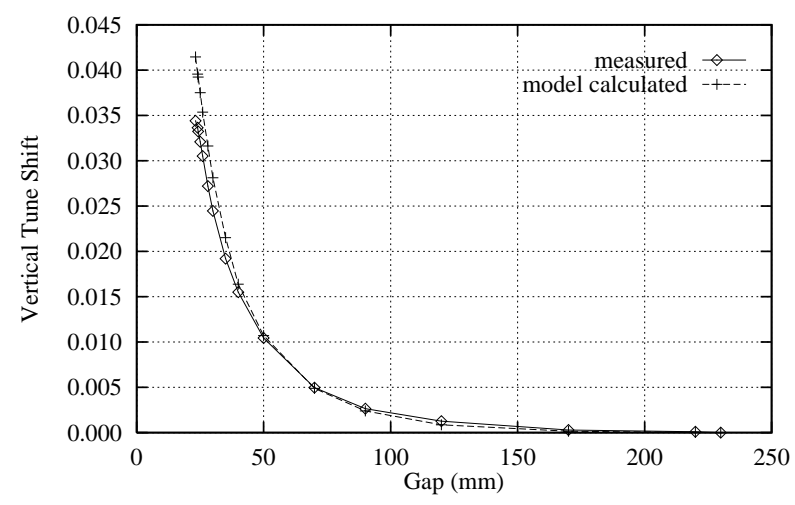

Figure 1: The measured and observed vertical tune shift v.s. wiggler gap.

The betatron function in each quadrupole location was measured with the trim coil in the quadrupole magnet. The measured vertical optics and beta-beat at wiggler gap $23.2 \mathrm{~mm}$ are shown in Fig. 2 and 3. Excellent agreement between measured vertical beta-beat and model calculated values are found. In the horizontal plane, the optics is essentially no change. To correct the optics and tune shift with local quadrupoles require some changes in quadrupole strengths. However, quadrupole trim is not large enough and independent quadrupole is not available in the time being.

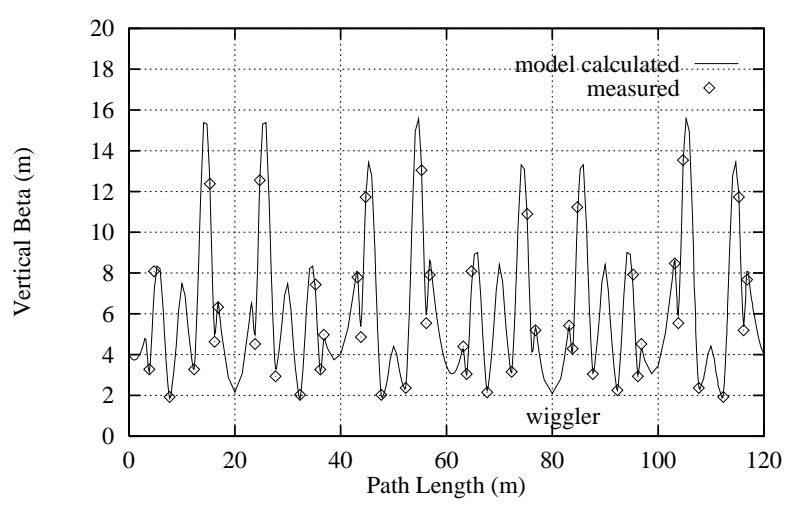

Figure 2: Measured and model calculated vertical betatron function with wiggler gap closed to $23.2 \mathrm{~mm}$. The wiggler center is at $80 \mathrm{~m}$ from the injection point.

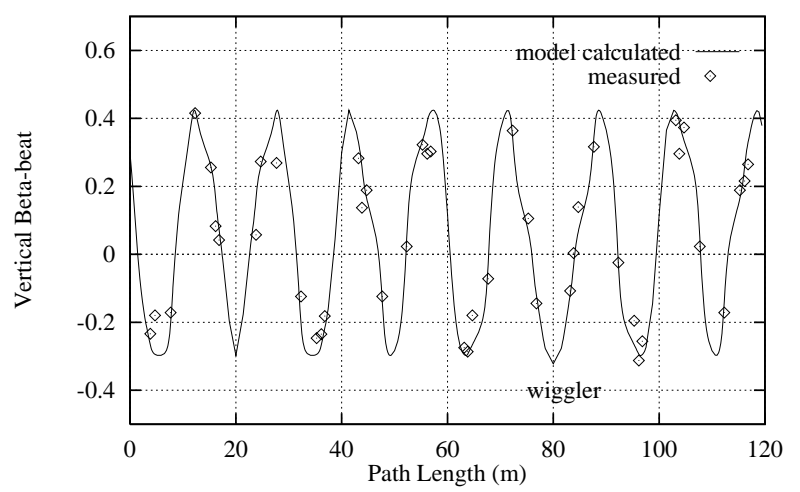

Figure 3: The measured and model calculated beta-beat between no wiggler and with wiggler gap $23.2 \mathrm{~mm}$.

The dynamic aperture, either in the horizontal or vertical plane, is measured and compared between open and close gap. The lifetime of the ring was measured at different scraper setting, which located at upstream long straight section. The physical half aperture of the wiggler chamber is $40 \mathrm{~mm} \times 8.5 \mathrm{~mm}$. There was a slight shrinkage of the dynamic aperture in both planes when the wiggler gap was closed as shown in Fig. 4 and 5. The increase of the sextupole strength to damp the transverse beam oscillation causes larger reduction of dynamic aperture by $1 \mathrm{~mm}$ or so in Fig. 6.

The integrated field strength was corrected by the end correctors as a function wiggler gap in order to reduce the orbit change locally and globally. However, we observed a significant change in the vertical orbit which increased when gap became smaller and the error source was traced to be at wiggler position. We re-established a follow gap end corrector setting table and the orbit change has been reduced to some extent as shown in Fig. 7.

\section{ACKNOWLEDGEMENT}

The authors would like to thank the help from Wiggler Task Force members C.H. Chang, T.C. Fan, G.F. Lin, C. H. Lee and Control Group member J. Chen, as well as all Technical staff. 


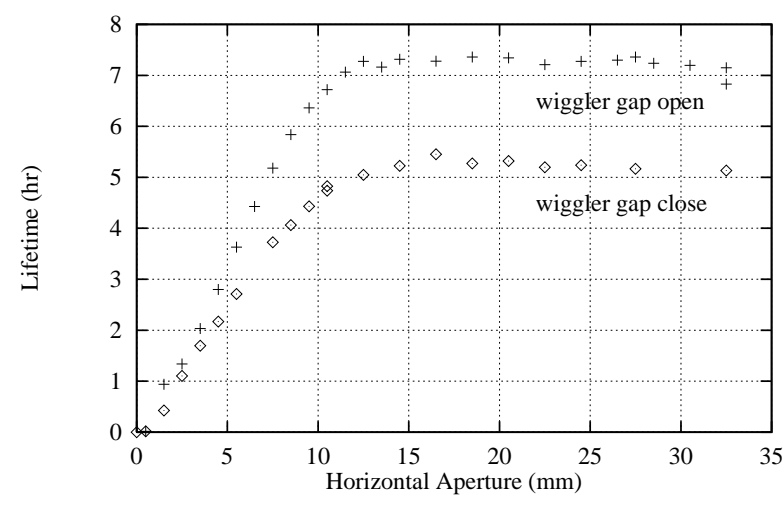

Figure 4: Horizontal dynamic aperture measurement with wiggler fully open and closed to $23.2 \mathrm{~mm}$ with $\mathrm{SD}=107.8 \mathrm{~A}$ and $\mathrm{SF}=91.8 \mathrm{~A}$. The chromaticities are near zero.

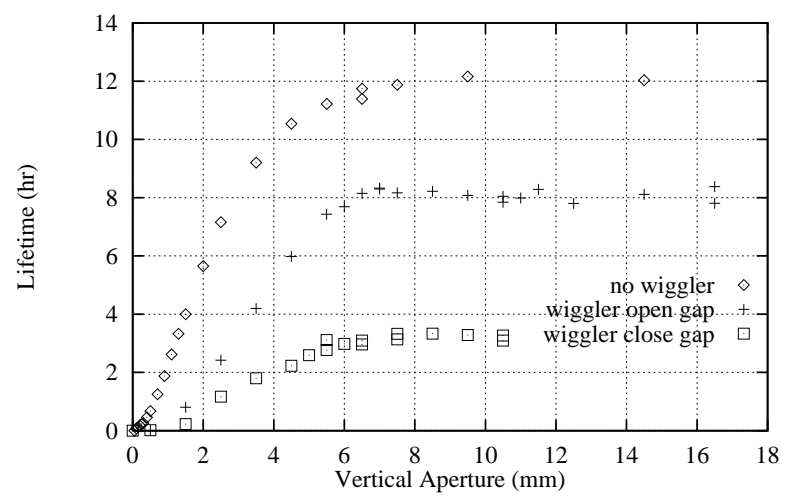

Figure 5: Vertical dynamic aperture measurement with wiggler fully open and closed to $23.2 \mathrm{~mm}$ with $\mathrm{SD}=107.8 \mathrm{~A}$ and $\mathrm{SF}=91.8$ A. In addition, a measurement without wiggler is also shown.

\section{REFERENCES}

[1] E. Yen, "Present Status of SRRC", 1993 IEEE PAC Conf. Proc., p. 1460.

[2] Y.C. Liu, "The Status of SRRC", 1994 EAPC Conf. Proc., p. 110.

[3] W.K. Lau et al., "Study of Longitudinal Coupled Bunch Instabilities in the SRRC Storage Ring", these proceedings.

[4] G.J. Jan et. al., "Bunch Killer System at SRRC", these proceedings.

[5] K.T. Hsu et. al., "Damping Rates of the SRRC Storage Ring", these proceedings.

[6] C.C. Kuo, et. al., "Closed Orbit Measurement and Correction of the SRRC Storage Ring", 1994 EPAC Conf. Proc., p. 1018.

[7] W.T. Weng et. al., "Beam Stability at SRRC Storage Ring", these proceedings.

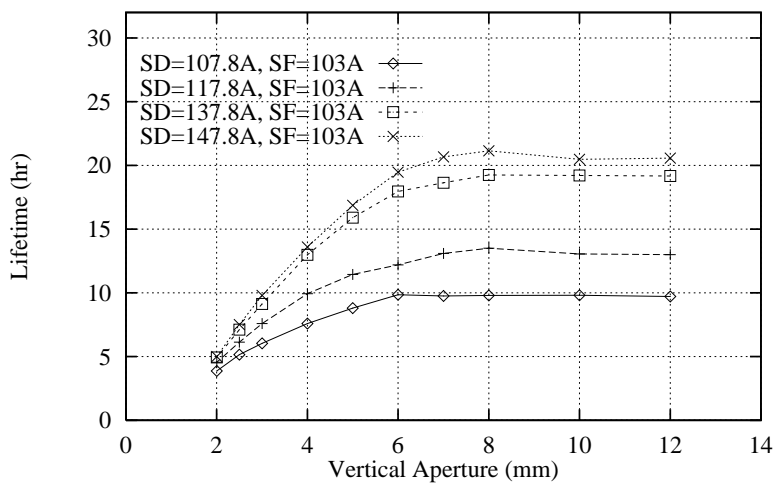

Figure 6: Vertical dynamic aperture measurement at different defocusing sextupole setting ( $\mathrm{SF}=103 \mathrm{~A}$ ) with wiggler gap fully open. The increase of lifetime with smaller sextupole setting is due to increase of Touschek lifetime of the unstable beam.

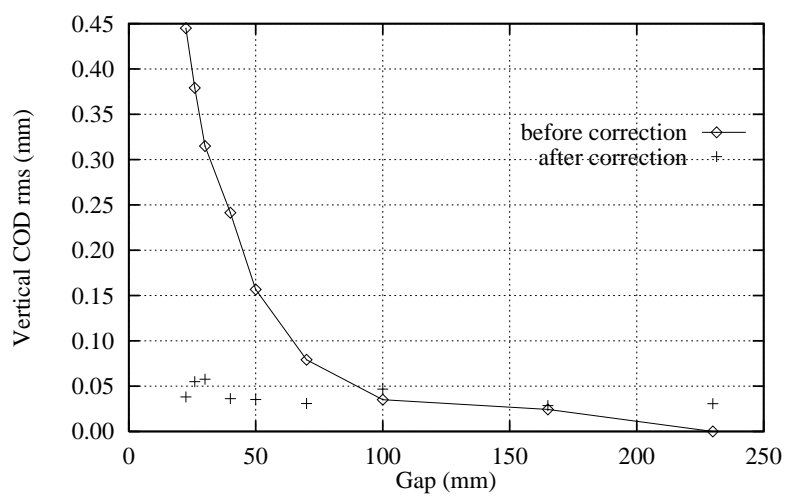

Figure 7: Vertical rms closed orbit change before and after end corrector modification v.s. wiggler gap. 